\title{
Trends in Agricultural Production: Microencapsulation
}

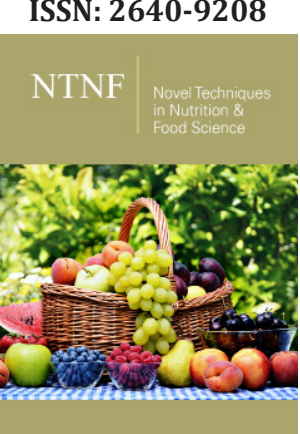

${ }^{* 1}$ Corresponding author: Marko Vinceković, Department of chemistry, Croatia

Submission: 眥 August 01, 2019

Published: 眥August 13, 2019

Volume 4 - Issue 2

How to cite this article: Marko $\mathrm{V}$, Vlahoviček K K, Slaven J. Trends in Agricultural Production: Microencapsulation. Nov Tech Nutri Food Sci. 4(2).NTNF.000583.2019.

DOI: $10.31031 /$ NTNF.2019.04.000583

Copyright@ Marko Vinceković, This article is distributed under the terms of the Creative Commons Attribution 4.0 International License, which permits unrestricted use and redistribution provided that the original author and source are credited.

\author{
Marko Vinceković*, Vlahoviček Kahlina K and Slaven Jurić \\ Department of chemistry, Croatia
}

\section{Abstract}

Trends in microencapsulation application in agriculture are focused on the preparation of complex biopolymer based microparticles, i.e. preparation of microspheres or microcapsules simultaneously loaded with chemical and biological agents to achieve a triple effect: protection, nutrition and production of plants fortified with important bioactive compounds.

Keywords: Encapsulation; Agriculture; Protection; Nutrition; Agroecological; Time-release mechanism

\section{Introduction}

The use of agrochemicals in plant productions have significant consequences for the environment and human health because most of them are persistent organic pollutants. Agrochemicals are useful and legitimate tools that can provide significant benefits to our society, but we need to use them with reduced environmental impact. Worldwide intention to diminish the use of agrochemicals is focused on more environmentally safe agroformulations. A much better understanding of the principles of colloid and surface chemistry in recent decades has led to the development of a new generation of agro-formulations (suspension concentrate, water-dispersible granules, capsule suspensions, etc.) for applying agrochemicals in a safer and more efficient way [1]. Process of bioactive agents loading in microparticles (microspheres or microcapsules) has been developed in recent years as an advanced tool for ecological and sustainable plant production. Encapsulation in biopolymer matrices has been recognized as an effective method for controlled release of a bioactive agent used for plant protection.

The benefits conferred by encapsulation include the slow release of the bioactive ingredient, more efficient exploitation of the chemical, greater safety to the user, and better protection of the environment. Encapsulation is a process of a substance (called as active agent or ingredient, core material, fill, payload, nucleus or internal phase) loading in particles ranging from nano to micron size [2]. The materials encapsulating an active agent usually used in agriculture are biopolymers. They are biodegradable, abundant in nature, renewable, nontoxic and relatively cheap. Another important property of biopolymers is a high content of functional groups including hydroxyl, amino, and carboxylic acid groups allowing tailoring of their properties by changing the physicochemical experimental conditions (e.g. media composition, temperature, and $\mathrm{pH}$ ). Microencapsulation is one of the most intriguing fields in the area of the active agent's delivery systems. It demands an interdisciplinary approach that requires fundamental investigation in various scientific fields and to an in-depth understanding of active agent's stabilization. Microparticle with an active agent should be designed to extend the activity, reduce toxicity, evaporation, and leaching as well as to diminish active agent amount required for the application. Key functionality that can be provided by encapsulation has controlled the release of a bioactive agent at the right place and the right time. Biopolymer based microparticles with a single bioactive agent have extensive applications in agriculture and became one of the standard agro-formulation [3]. Up till now, various crop protecting agents were encapsulated, e.g. pesticides, insecticides, fungicides. The benefits by encapsulation include more efficient exploitation, greater safety to the user and better protection of the environment.

Trends in microparticle application in agriculture are focused on the preparation of complex biopolymer-based microparticles, i.e. preparation of microparticle formulations involving several active agents. Intensive research in recent years has provided many 
new insights into processes and mechanisms of intermolecular interactions in biopolymer-based microparticles however our knowledge of simultaneous encapsulation of biological and chemical agents is still rather limited. Despite the array of encapsulation methodologies for encapsulation of one bioactive agent, there are only a few data in the literature about simultaneous encapsulation and delivery of biological and chemical agents with the role for plant protection and nutrition [4]. The requirement for simultaneous loading is to choose a material which can incorporate both, chemical (various micro and macronutrients) and biological (mainly bacteria, nematodes, and fungi) agents, in the same compartment without diminishing their activities. Thinking about potential agricultural applications, this is not only a demand but also a scientific challenge.

Investigations of intermolecular interactions and structure/ properties relationship in biopolymer matrices loaded with biological and chemical agents enhance our ability to control their release behavior and may aid in developing new microparticles to tailor their properties according to the different needs of various applications. By adopting a systematic formulation approach, an optimum effect can be reached to achieve prolonged protection and nutrition effects on the plants over the plant maturation time. Recently, we have applied variously designed microparticles simultaneously loaded with chemical and biological agents on several plants under greenhouse conditions and in an open field. After the treatments, grapevine vine leaves reached a significant increase in bioactive potential compared to the control [5]. This important finding indicates besides plant nutrition and protection, microparticles loaded simultaneously with chemical and biological agents can increase the production of plant secondary metabolites, that is the production of plants with improved nutritional quality as potential dietary sources of important bioactive compounds. Production of functional foods with bioactive compounds is steadily increasing as a result of the increased need to use natural resources in nutrition and prevention of various chronic diseases and health care.

Bioencapsulation alongside the presence of chemical agents revealed to be a sustainable, environmentally friendly, rapid, convenient, economical and efficient for targeted delivery of bioactive agents for plant nutrition, protection and functional food production. This offers great perspectives to enhance existing plants production, which could not be incorporated within classical formulations.

\section{References}

1. Rodkham DK (2000) Colloid and interface science in formulation research for crop protection products. Current Opinion in Colloid Interface Science 5(5-6): 280-287.

2. Thies C (2006) Microencapsulation. In: Seidel A (Ed.), Kirk-Other Encyclopedia of Chemical Technology, $5^{\text {th }}$ edn, Wiley, Hoboken, New Jersey, USA, pp. 438-463.

3. Nuruzzaman M, Rahman MM, Liu Y, Naidu R (2016) Nanoencapsulation, nano-guard for pesticides: a new window for safe application. Journal of Agricultural and Food Chemistry 64(7): 1447-1483.

4. Vinceković M, Jalšenjak N, Pintarić ST, Đermić E, Bujan M, et al. (2016) Encapsulation of biological and chemical agents for plant nutrition and protection: Chitosan/Alginate microcapsules loaded with copper cations and Trichoderma viride. J Agric Food Chem 64(43): 8073-8083.

5. Vinceković M, Bandić LM, Jurić S, Jalšenjak N, Čaić A, et al. (2019) The enhancement of bioactive potential in Vitis vinifera leaves by application of microspheres loaded with biological and chemical agents. Journal of Plant Nutrition 42: 543-558. 